\title{
Difficult paediatric airway
}

\author{
Mogane P \\ Department of Anaesthesia, Chris Hani Baragwanath Academic Hospital,University of the Witwatersrand, South Africa \\ Corresponding author, email: moganep@gmail.com
}

\section{Introduction}

Evidence from the ASA Closed Claim Database shows that respiratory events are more common in children than adults ( $43 \%$ vs $30 \%$ respectively; $p \leq 0.01$ ) with the commonest cause of morbidity and mortality due to the inability to ventilate rather than to intubate. ${ }^{1,2}$ Contributing factors are the anatomical and physiological differences which exist between the neonate, infant, child and adult. ${ }^{3}$ These make the normal paediatric airway potentially difficult particularly in children under the age of one year. Repeated attempts at securing the airway may potentiate tissue trauma, bleeding and mucosal oedema which can transform an airway that can be ventilated into one that cannot (cannot intubate, cannot oxygenate situation). The difficult airway in the paediatric population is a rare (incidence of about $0.15-1.4 \%)^{4}$ and challenging entity, which is often devastating if inappropriately managed.

A difficult airway is defined as a 'clinical situation in which a conventionally trained anaesthesiologist experiences difficulties with facemask ventilation, tracheal intubation or both'. The recommendation is that subsequent attempts use alternate devices. This represents a complex interaction between patient factors, the clinical setting and the skills of the practitioner. ${ }^{5}$

The 1993 ASA guidelines defined a difficult intubation as 'when proper insertion of the tracheal tube with conventional laryngoscopy requires more than three attempts or more than 10 min'. $^{6}$ The 2013 updated report has revised this, and now defines difficult tracheal intubation simply as 'requiring multiple attempts'. Difficult facemask or supra-glottic ventilation is defined as an inability to provide adequate ventilation because of inadequate seal, leak, or excessive resistance to the ingress or egress of gas. ${ }^{5}$ An alternate definition of difficult mask ventilation, is the inability of an unassisted anaesthesiologist to maintain the measured oxygen saturation as measured by pulse oximetry $>92 \%$ or to prevent or reverse signs of inadequate ventilation during positive pressure ventilation under general anaesthesia. ${ }^{7}$ The incidence of unexpected difficult bag mask ventilation may be as high as $6 \%{ }^{4,8}$

\section{Guidelines}

In 2012, the Guidelines Group, supported by the Association of Paediatric Anaesthetists of Great Britain and Ireland, the Difficult Airway Society and liaising with the Royal College of Anaesthetists developed guidelines following an exhaustive process which involved a Delphi analysis and extensive literature review. They found that there was little grade 1 evidence to support good practice in the management of the difficult paediatric airway, and guidance must therefore be essentially a clinical issue. The target audience for these guidelines is the anaesthetist working in the non-specialist paediatric setting who wishes to learn or maintain paediatric airway skills, rehearse unexpected difficult airway scenarios and teach good practice.8,9 Three algorithms were chosen based on the clinical scenarios that would benefit most from the straightforward plans:

- Difficult mask ventilation (MV) - during routine induction of anaesthesia in a child aged 1 to 8 years.

- Unanticipated difficult tracheal intubation $(\mathrm{TI})$ - during routine induction of anaesthesia in a child aged 1 to 8 years (Figure 1).

- Cannot intubate and cannot ventilate (CICV) in a paralysed anaesthetised child aged 1 to 8 years.

The assumptions made are that children older than eight years of age could use the adult Difficult Airway Society (DAS) guidelines; anticipated airways are predictable and therefore can be adequately prepared for; and more importantly that practice guidelines are systematically developed recommendations that assist the practitioner and patient in making decisions. They may be adopted, modified or rejected according to clinical needs and constraints and are not intended to replace local institutional policies. ${ }^{5}$ The question then is do we have local institutional policies on the management of paediatric airways?

It has been recognised that factors influencing performances can be broadly divided into those relating to preparation (guidelines, training, experience, consultation and planning) and those relating to implementation (impaired decision making fixation, omission or failure to act and the inability of teams to function effectively) of the airway plan.

The Vortex (Figure 2) was thus developed as a cognitive tool that may be effective in reducing implementation errors of the difficult airway algorithms. The information-dense, text-based presentation of the major airway algorithms, despite being technically accurate, makes it more strenuous for teams to use the information within them during the stress and uncertainty of an evolving airway emergency. A simple, low-content, contextindependent, predominantly graphic design of the Vortex 


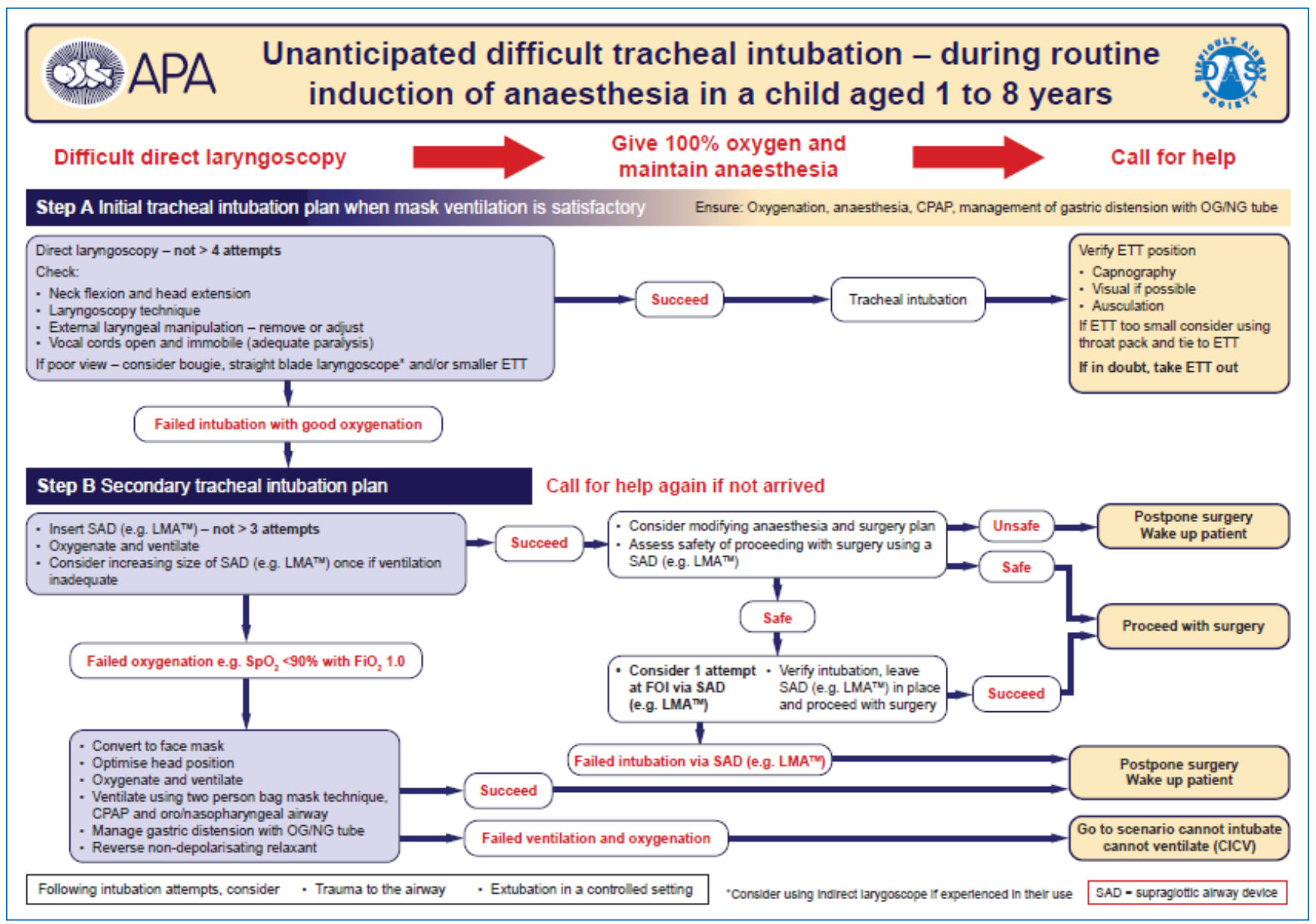

Figure 1. Unanticipated difficult tracheal intubation during routine induction of anaesthesia in a child aged 1 to 8 years

\section{The Emergency Airway Cognitive Tool}
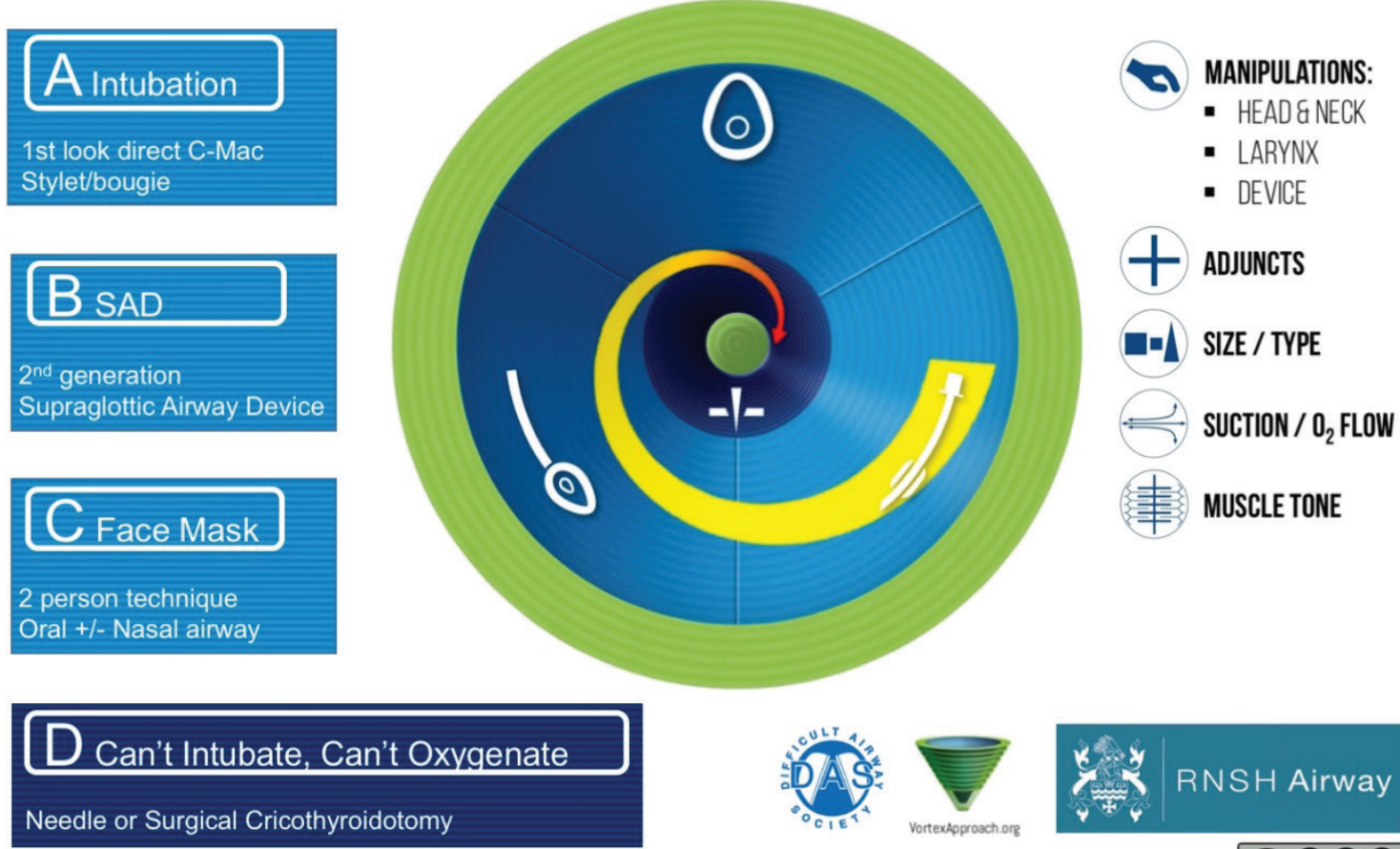

RNSH Airway

Adapted from Difficult Airway Society guidelines 2015 and the Vortex approach $\odot$ Nicholas Chrimes 2013, 2016 (used with permission)

(c) (1) $\Theta \Theta$

Figure 2. Vortex implementation tool (superior and lateral aspects)

Green zone - adequate alveolar oxygen delivery ${ }^{11}$ 
approach makes it more suited to real-time use during crisis situations irrespective of the clinical background of the user. ${ }^{10}$

Usage is based on the principle that there are three none surgical techniques to establish a patent airway: face mask, a supraglottic device, or an endotracheal tube. Inability to achieve adequate alveolar oxygenation after a best effort at any or all of the three lifelines mandates spiral movement inwards by initiating the can't intubate can't oxygenate (CICO) rescue plan, i.e. surgical airway. Any attempt at a lifeline must incorporate optimisation that has previously not been implemented in the form of manipulations (of head, neck, larynx or device), usage of adjuncts, changing sizes and types of device, usage of suction and supplemental oxygen and ensuring that airway muscle tone is not a hinderance. The Vortex should not be considered an alternative to the existing algorithms, but rather a complementary tool intended to place emphasis on a shared, simplified mental model enhancing situational awareness, team performance and minimising error. ${ }^{10}$

\section{Literature around the paediatric airway}

In 2011, the Fourth National Audit Project (NAP4) of the Royal College of Anaesthetists and the Difficult Airway Society was established with the aim of estimating the incidence of and contributing factors to major complications of airway management during anaesthesia in the United Kingdom. Some of the significant findings were: (i) more than half the patients were ASA 1-2, during elective surgery under the care of anaesthetic consultants, (ii) aspiration was the most frequent cause of mortality, (iii) many reports showed evidence of poor planning of primary and subsequent rescue techniques, (iv) cricothyroidotomy by anaesthetists was associated with high rate of failure, $(v)$ omission or incorrect interpretation of capnography led to undiagnosed oesophageal intubation, (vi) elements of poor management were observed in the majority of airway complications and most deaths. Thus, there was a significant 'human factor' contribution. ${ }^{12}$ Several system recommendations were made. These included development of local intubation algorithms modified from DAS' plan with clear pathways for airway escalation, standardisation of approach and equipment, universal use of the pre-intubation checklist, universal use of the end-tidal capnography to confirm correct endotracheal placement and multidisciplinary training (anaesthesia, ED and ICU) in airway technical and nontechnical skills.

Secondary analysis of airway and ventilation management of the Anaesthesia Practice in Children Observational Trial (APRICOT) looked primarily at the incidence of difficult airway management, and secondarily at the associations between difficult airway management, known pre-existing respiratory risk factors and the occurrence of critical respiratory events. There was a strong association between severe respiratory critical events and the number of attempts to secure the airways, the airway management device, the presence of preoperative respiratory risk factors and young age (neonates and children $<1$ year of age). Multiple tracheal intubation attempts were reported in the presence of $\mathrm{CL}$ grades 1 and 2, which may reflect on planning and practitioner technique. The use of video laryngoscopy was surprisingly low, indicating a poor availability of the device or a principle use as a rescue tool during unexpected difficult tracheal intubation ( $\mathrm{TI}$ ). These last 2 points (difficult $\mathrm{TI}$ with $\mathrm{CL}$ grade 1 and 2 , and low use of $\mathrm{VL}$ ) are in stark contrast to the findings of the PeDI study. ${ }^{13}$

While APRICOT was enrolling patients in Europe, the Pediatric Difficult Intubation (PeDI) registry was collecting data on > 2000 difficult airway events from the USA. Both APRICOT and PeDI found an increased likelihood of airway difficulty in younger children (particularly $<1$ year of age). They also noted significantly increased respiratory events in patients with 3 or more attempts at intubation, suggesting the morbidity may be decreased by reducing the total number of tracheal intubation attempts.

The multicentre PeDI registry tried to characterise risk factors for difficult Tl, establish success rates of various techniques, catalogue the complications associated with difficult $\mathrm{Tl}$, and establish the effect of more than $2 \mathrm{TI}$ attempts. The most frequently attempted first TI techniques were direct laryngoscopy (46\%), FOB (28\%) and indirect video laryngoscopy (18\%), with first attempt success rates of $3 \%, 54 \%$ and $55 \%$ respectively. The most severe complication was cardiac arrest (which occurred in $2 \%$ ). Temporary hypoxaemia was the most frequent nonsevere complication. Interestingly oxygen was rarely given during tracheal intubation attempts. $80 \%$ of difficult tracheal intubations were anticipated which allowed for appropriate planning. The occurrence of complications was associated with more than $2 \mathrm{Tl}$ attempts, a weight $<10 \mathrm{kgs}$, short thyromental distance, abnormal airway physical examination, and 3 direct laryngoscopy attempts before an indirect technique. ${ }^{14}$ Suggestions from the study were:

1. Practitioners should 'actively pursue opportunities to deliver supplemental oxygen throughout the process of difficult airway management. Preoxygenation is practised but few (10\% in PeDI registry) oxygenate during the attempt. When hypoxaemia occurs during TI the intubation attempt is interrupted to ventilate and oxygenate the patient. Passive oxygenation should be instituted.

2. Derive and implement a standardised airway checklist.

3. Transition to the most experienced clinician should happen quickly.

4. Use extraglottic airway device early.

A sub-analysis of the PeDI registry looked at video laryngoscopy (VL) versus fibreoptic bronchoscopy (FOB) intubation through a supraglottic airway device (SAD) in children with a difficult airway. The main finding was that FOB-SAD and VL have similar rates of first attempt success in children with difficult airways. However, FOB-SAD as the first technique was associated with significantly fewer intubation attempts and changes in airway management strategies, as demonstrated by a higher overall success rate. Furthermore, in infants $<1 \mathrm{yr}$, FOB-SAD had a significantly higher success rate (54\%) compared to VL (36\%). Hypoxaemia was significantly less common during FOB-SAD technique when 
continuous ventilation was used throughout the intubation attempt. Where both these techniques failed, tracheal intubation was eventually achieved with $D L$, FOB without SAD, flexible FOB with video laryngoscopy, rigid bronchoscopy and very rarely a surgical airway was required. ${ }^{15}$

Some recommendations are that the paediatric specific guidelines from DAS should be updated to emphasise:

1. Children $<10 \mathrm{~kg}$ represent a high risk population. In this population supplemental oxygen should be considered.

2. Direct laryngoscopy (DL) should be limited to less than 2 attempts with an early transition to an advanced alternative airway technique (e.g. VL, FOB with or without SAD).

3. A SAD should be readily available and inserted if difficult ventilation is encountered, or if rescue oxygenation is needed.

4. Structured teaching and practice (e.g. simulation) with advanced airway devices should take place in normal patients. ${ }^{16,17}$

\section{Approach to difficult airway ${ }^{18}$}

\section{Assessment}

Recognising the patient with a potentially difficult airway is an opportunity that allows you to prepare ahead with appropriate equipment, personnel and a backup plan. Many methods exist for evaluating and predicting difficult airway in adults; no comparable methods have been forthcoming in children. Characteristics that may warn one of a difficult laryngoscopy and intubation include (Table 1):

- specific syndromes

- maxilla-facial malformations

- anomalies of the mouth, tongue or teeth

- cervical spine pathologies
- oropharyngeal space occupying lesions 19

- previous history of difficult airway.

The lower airway passages can be accessed via the mouth, nose or neck. The most viable options should be reviewed. Thereafter can you align the 3 airway axes (oral, pharyngeal and laryngeal axes) sufficiently to bring the larynx into view if direct laryngoscopy is the plan of choice. Perhaps even more important than recognising a challenging intubation, is identifying the patient who will be difficult to ventilate

\section{Teamwork and communication}

Key factors in making problem solving and crisis management successful is teamwork and communication. One needs to take stock of the situation and self, there needs to be a co-ordinated team effort with a shared mental model. Everyone needs to know what the problem is, the intended plan in resolving it and what their individual role is going to be. As part of the team, there needs to be an individual dedicated to situational monitoring i.e. patient condition, vitals and time lapse during attempts.

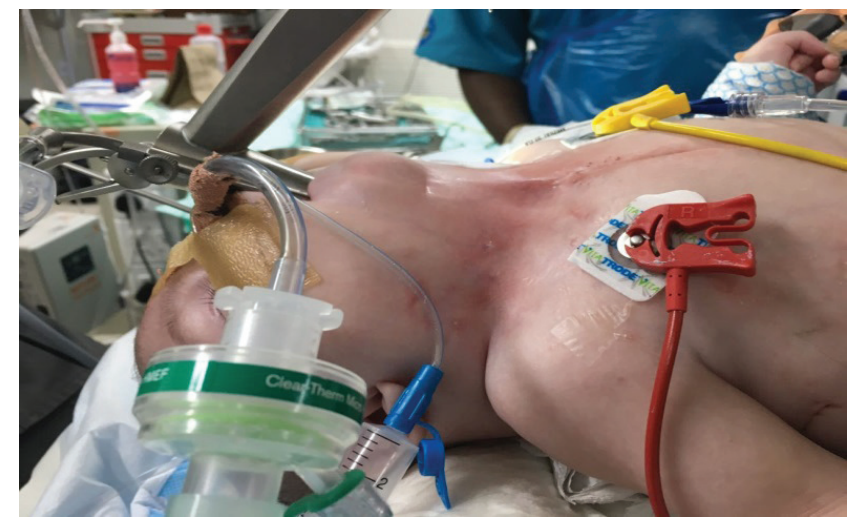

Figure 3. Patient for cardiac surgery with undiagnosed syndrome with features suggestive of difficult airway

Table 1. Predictors of an expected difficult airway $y^{4,19}$

\begin{tabular}{|c|c|}
\hline \multicolumn{2}{|c|}{ Predictors of an expected difficult airway } \\
\hline Soft tissue pathologies & $\begin{array}{l}\text { Tumours } \\
\text { Abscess } \\
\text { Scars } \\
\text { Post radiation therapies } \\
\text { Burns, trauma }\end{array}$ \\
\hline Maxilla-facial malformations & $\begin{array}{l}\text { Hypoplasia of the mandible } \\
\text { Retrognatia/micrognatia } \\
\text { Facial asymmetries (including ear anomalies) } \\
\text { Mandibular joint ankyloses (reduced mouth opening) }\end{array}$ \\
\hline Mouth, tongue and teeth anomalies & $\begin{array}{l}\text { Microstomia } \\
\text { Macroglossia } \\
\text { Cystic hygroma } \\
\text { Overbite }\end{array}$ \\
\hline Cervical spine pathologies & $\begin{array}{l}\text { Reduced movement, e.g. Klippel-Feil syndrome } \\
\text { Instability, e.g. Downs syndrome, rheumatoid arthritis, mucopolysaccaridosis }\end{array}$ \\
\hline Airway obstruction & $\begin{array}{l}\text { Obstructive sleep apnoea } \\
\text { Stridor } \\
\text { Infective processes, e.g. epiglottitis, laryngotracheitis, abscess } \\
\text { Bleeding post tonsillectomy } \\
\text { Anaphylaxis } \\
\text { Airway foreign body }\end{array}$ \\
\hline
\end{tabular}

Specific syndromes, e.g. Pierre Robin, Treacher Collins, Goldenhar

History of difficult airway 
Careful documentation of all measures taken during the airway management should be detailed in file, and patient or parents notified of pertinent facts. ${ }^{18}$

\section{Planning (PREPARE FOR FAILURE)}

Intubation is usually uneventful, thus we are lulled into a false sense of security. Take no intubation for granted, always prepare for failure! Realistically assess if you are the optimal person or institution to manage the airway. You may have to refer to a specialised unit with appropriate equipment and capability for support from the otorhinolaryngologist (ENT surgeon). Ensure intravenous access is well secured and working prior to embarking on the difficult airway, where necessary an intraosseous line may be an alternative. Position is critical, ensure it is optimised before starting.

The first choice in the management of a potentially difficult airway, whether the child is sedated or under general anaesthesia, is to maintain spontaneous ventilation. The reasons for maintaining spontaneous gaseous exchange are that neuromuscular blockade may result in total airway obstruction owing to loss of tone of the tongue, pharyngeal and laryngeal muscles and suspensory ligaments which may not be alleviated by manual ventilation. Secondly, if a child is paralysed, the loss of spontaneous breathing leads to earlier onset of apnoea and hypoxemia, and it eliminates a technique useful in locating the glottis (see 'following the bubble'). There has been some shift in practice to paralysing children with difficult airways with non-depolarising muscle relaxants where it is felt that muscle tone is the major contributor to inability to visualise and intubate the airway, or where there is a fear of airway activation (laryngospasm, bronchospasm, coughing). This can only occur after confirming easy mask ventilation. Rocuronium is often used due to the availability of sugammadex to reverse the paralysis should the emergency situation arise. ${ }^{14,20}$

A simplified approach to the airway should be outlined as in Table 2. This is not prescriptive as to which specific technique should be employed with each attempt, rather the focus is on preparation, assessment, team skills and communication of the airway plan between the team and team leader, and the avoidance of fixation error. ${ }^{21}$
Table 2. Simplified airway algorithm ${ }^{2}$

\begin{tabular}{l} 
PREPARATION - ASSESS, CHECK, PLAN, OPTIMISE \\
\hline PLAN A - INITIAL INTUBATION STRATEGY \\
- direct or indirect laryngoscopy, e.g. video laryngoscopy assisted by \\
usage of bougie, stylet or wand \\
PLAN B - SECONDARY INTUBATION STRATEGY \\
- fibreoptic bronchoscopy \pm supraglottic airway device \\
- rigid bronchoscopy \\
PLAN C - MAINTAIN OXYGENATION \& VENTILATION \\
- facemask with oropharyngeal or nasopharyngeal airway \\
- supraglottic airway device \\
- high flow nasal cannula or modified Trumpet manoeuvre \\
PLAN D - RESCUE TECHNIQUE FOR 'CAN'T INTUBATE, CAN'T \\
VENTILATE \\
- needle or scalpel cricothyroidotomy \\
- surgeon prepped and ready for surgical airway \\
\hline
\end{tabular}

Stressful situations make even the most skilled providers forget crucial steps. Checklists help to eliminate the weaknesses of human factor. The Difficult Airway Society has an intubation checklist developed for use in the critically ill adult patient which can be sampled and modified for use in the paediatric population (Table 3).22

\section{Preparation (equipment)}

A wide array of equipment is available, however resources and skill may restrict what may be used to rescue the airway. Have a prepared difficult airway trolley with all the necessary equipment in a central area. There should be knowledge of many alternative intubation and ventilation equipment as each has limitations depending on the clinical scenario, e.g. high rates of first pass failure using video laryngoscopy include blood in airway, limited mouth opening, airway oedema or a mass. Practise with your devices during routine intubations to become proficient; this encourages competency with what is available. Remember it is the operator's expertise rather than a certain technique or equipment which leads to successful management.

Based on the literature published, video laryngoscopy (VL) and fibreoptic bronchoscopy (FOB) have revolutionised the management of the difficult airway and are accepted as primary techniques. ${ }^{14,15}$ Supraglottic airway devices are perhaps the most important rescue devices for clearing an obstructed airway as well as providing a conduit for fibreoptic intubation, while

Table 3. Suggested intubation checklist ${ }^{21,22}$

\begin{tabular}{|c|c|c|}
\hline TEAM & EQUIPMENT & PATIENT \\
\hline 1. Verbalise indication for intubation & 1. Check equipment & 1. Airway assessment \\
\hline 2. Allocate roles & - tracheal tubes & 2. Optimise position \\
\hline - team leader & - oro- and nasopharyngeal airway & $-<1$ yr: towel under shoulders \\
\hline$-1^{\text {st }} / 2^{\text {nd }}$ intubator & - direct/indirect laryngoscope & - > 8 yr: towel under head \\
\hline - intubators' assistant & - suction & 3. Optimise preoxygenation \\
\hline - drugs administrator & - supraglottic device & -3 mins or $\mathrm{ETO}_{2}>85 \%$ \\
\hline - monitoring patient & - flexible fibreoptic & - consider other forms of supplemental \\
\hline - runner & - surgical access kit & oxygenation \\
\hline - who will perform surgical airway & 2. Apply monitors & 4. Optimise patient \\
\hline 3. Confirm intubation plan & $\left(\mathrm{SpO}_{2}\right.$, waveform $\left.\mathrm{ETCO}_{2}, \mathrm{ECG}, \mathrm{NIBP}\right)$ & - fluid/pressor/inotrope \\
\hline - PLAN A, B, C, D & 3. Check drugs & - aspirate NGT \\
\hline Who do we call for help? & - reliable IV/IO access & \\
\hline Time keeper & $\begin{array}{l}\text { - induction agent, relaxant, pressor/inotrope, } \\
\text { sedation }\end{array}$ & \\
\hline
\end{tabular}


delivering adequate anaesthesia and maintaining oxygenation. In particular the air-Q laryngeal airway offers many advantages over traditional laryngeal masks when used as a conduit in intubation.

Novel techniques to consider where FOB or VL are not available:

- Optimal external laryngeal manipulation is particularly helpful in children with immobile or short necks.

- 'Follow the bubble' - in the spontaneously breathing patient or even if apnoeic (may need to apply gentle chest compression) air bubbles at the glottis opening may assist in the visualisation of the airway.

- Shaping the endotracheal tube into a 90-degree angle with a stylet, then placing the tip thereof behind the epiglottis (or the centre of the base of the tongues if the epiglottis is not visible) and then tracking the capnography as one blindly locates the glottis opening and trachea. ${ }^{20}$

- Retromolar, lateral or paraglossal approach using a straight blade $^{3,23}$ is particularly useful in children with a large tongue or small mandible.

- Blind nasal intubation.

\section{Don't forget the patient attached to the airway}

Consideration must be taken of the patient's pre-existing condition, e.g. many of the syndromic conditions have a coexisting cardiac lesion, and drugs given may render the child haemodynamically unstable. Designate an assistant solely tasked with monitoring and treating the patient while the airway is being dealt with. The speed of onset of critical hypoxia due to apnoea in children is faster. This can be prolonged by supplemental oxygenation. Routine preoxygenation must take place, followed by some passive (apnoeic) oxygenation techniques. This can be provided by:

- Nasal prong oxygen.

- High flow, positive-pressure, humidified oxygen via nasal cannula (e.g. Transnasal Humified Rapid Insufflation Ventilatory Exchange - THRIVE) at 1-2L/ $\mathrm{kg} / \mathrm{min}^{24}$
- Modified Trumpet manoeuvre (Figure 4) - insufflation into the hypopharynx via an endotracheal placed while other orifices not being used are occluded. ${ }^{25}$

- During FOB via SAD.

- Tracheal tube attached to a laryngoscope, e.g. Truview PCD videolaryngoscope. ${ }^{26}$

\section{Elective or emergency surgical access}

We all fear the catastrophic 'can't intubate can't oxygenate' scenario, yet we practise as though it will never happen to us. If an airway looks difficult, consider an awake intubation. Paediatric anaesthetists often avoid this due to fears of lack of patient cooperation, discomfort and lack of experience. Awake intubation with sedation is possible with proper preparation, well functioning intravenous access and where possible topicalisation of the airway. Topicalisation can take place via nebulised lignocaine; topical application of local anaesthetic sprays, jellies, or ointments; translaryngeal delivery of lignocaine; spray as you go through the suctioning channel of the FOB; or less rarely via a superior laryngeal nerve block. ${ }^{20}$

Important to note, sedate cautiously and be patient! Oversedation can lead to loss of airway muscle tone. It is best to rely on good airway local anaesthesia, rather than just sedation.

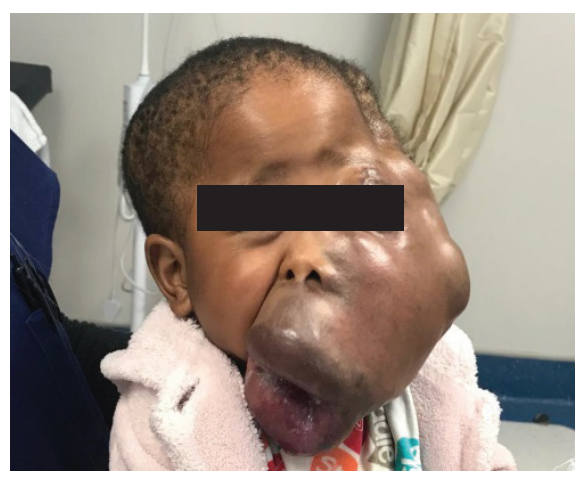

Figure 5. Patient with Sturge-Weber syndrome sedated with dexmedetomidine $3 \mathrm{ug} / \mathrm{kg}$ + ketamine $0.25-0.5 \mathrm{mg} / \mathrm{kg}$ IV followed by an awake tracheostomy

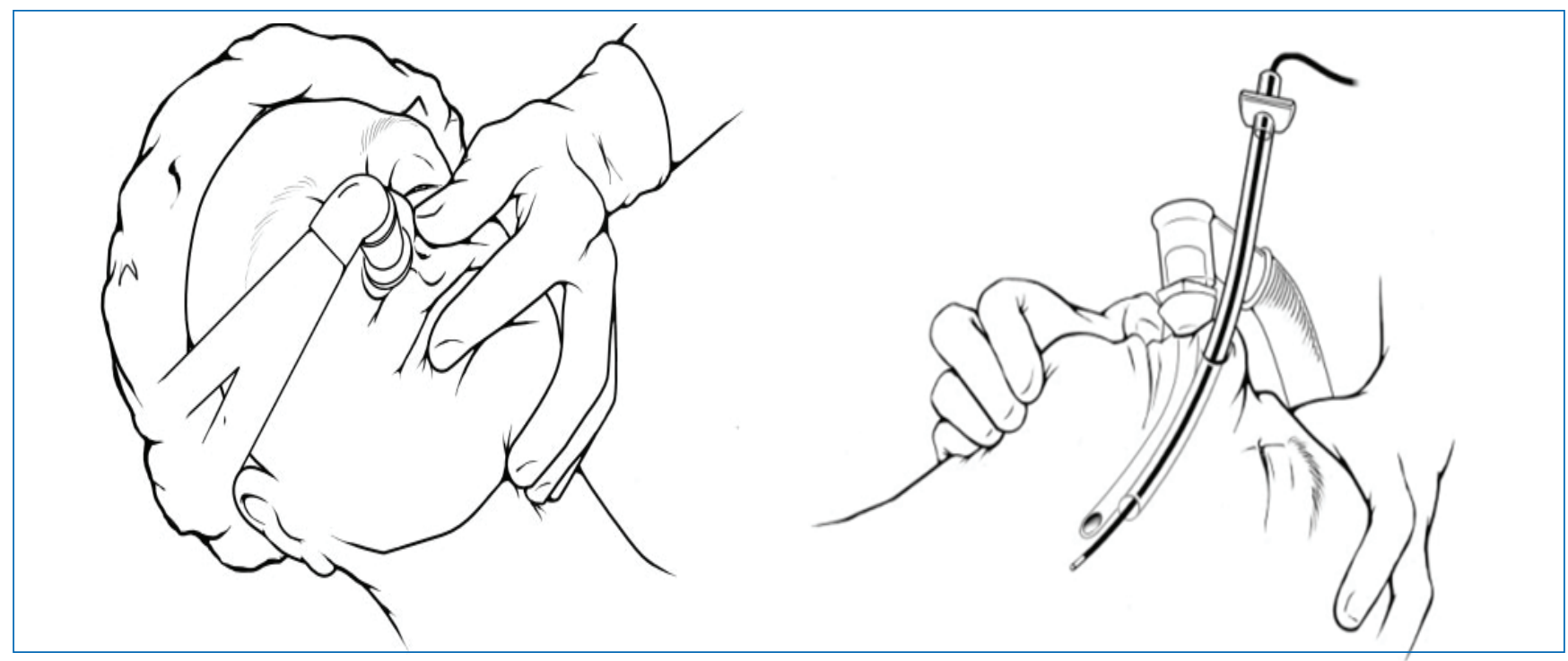

Figure 4. Modified Nasal Trumpet manoeuvre ${ }^{25}$ - controlled or assisted ventilation through the right nostril (with other orifices firmly occluded) while the other nostril is used for intubation via a fibreoptic scope 
Many alternatives exist with dexmedetomidine and ketamine having favourable outcomes.

The need for an emergency surgical airway in infants is very rare. There is a paucity of literature in this patient population and very little equipment developed in this area. Moreover, the cricothyroid membrane is difficult to identify and expeditious performance of a surgical airway is challenging even for the most skilled paediatric otolaryngologist. In a crisis, the fastest option to oxygenate is most likely through needle cricothyroidotomy (Figure 6). However this is performed with the risk of perforation of the posterior tracheal wall. ${ }^{17}$ There is currently insufficient evidence to prescribe one specific surgical airway technique over another, the biggest debate being whether to use a scalpel, a needle (cannula) cricothyroidotomy, a large bore catheter or the emergency cricothyroidotomy set. ${ }^{27}$ There is a suggestion that the current best practice in neonates and smaller children remains the needle cricothyroidotomy in the CICO situation ${ }^{17}$ due to the size of the cricothyroid membrane (CTM). Furthermore, in neonates and infants, full extension of the head and neck may not allow a flat enough approach to the CTM, and identification of landmarks may be technically difficult. Under these circumstances, a cannula tracheostomy may be necessary to avoid damage to the cricoid and thyroid cartilages. ${ }^{28}$ The APA Guidelines for the CICV paralysed child aged 1-8 years recommend the use of a cannula cricothyroidotomy/ tracheostomy. ${ }^{9}$ Evidence suggests that the more distal along the trachea front of neck access (FONA) is attempted, the lower the success rate for both cannula and scalpel techniques (i.e. cricothyroidotomy preferred to tracheotomy). In a semi-urgent scenario, ultrasound may be used to identify the CTM ${ }^{27}, 29$ (Figure 7 and 8). The appropriate equipment should ideally be prepacked and ready for use in the event of this much feared scenario.

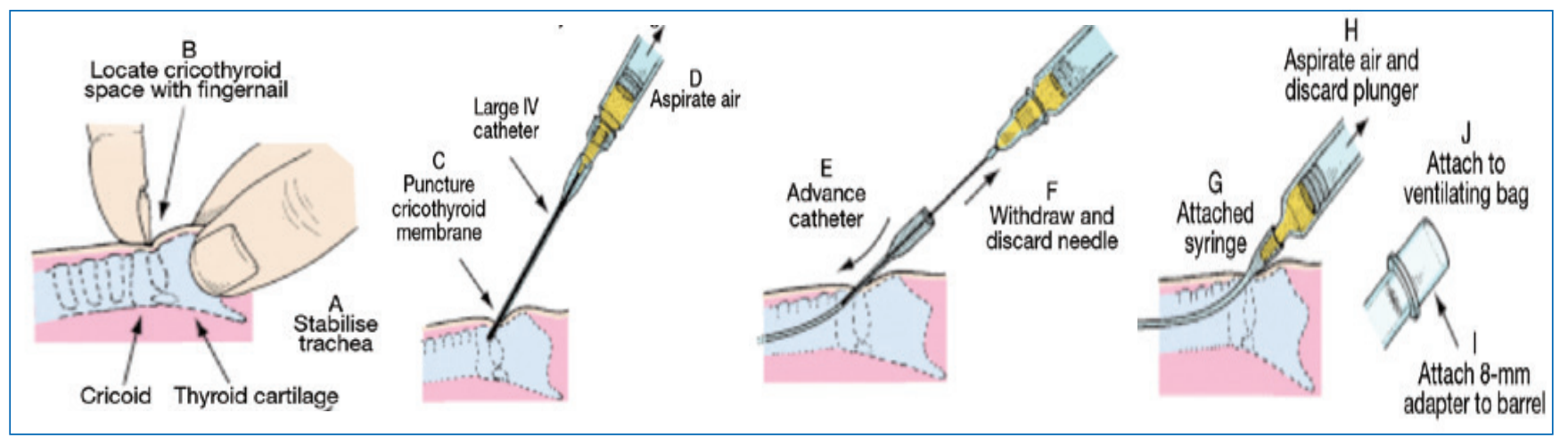

Figure 6. Technique of percutaneous needle cricothyroidotomy ${ }^{3}$

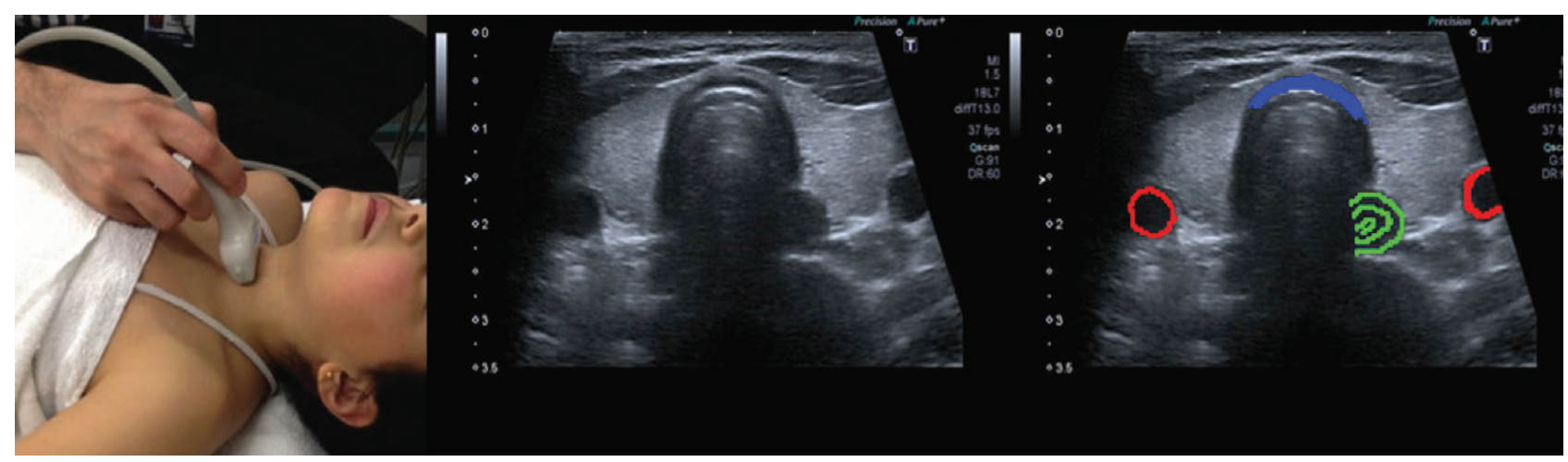

Figure 7. Ultrasound of transverse view of trachea

Tracheal ring (blue), oesophagus (green) and carotid arteries (red) ${ }^{29}$

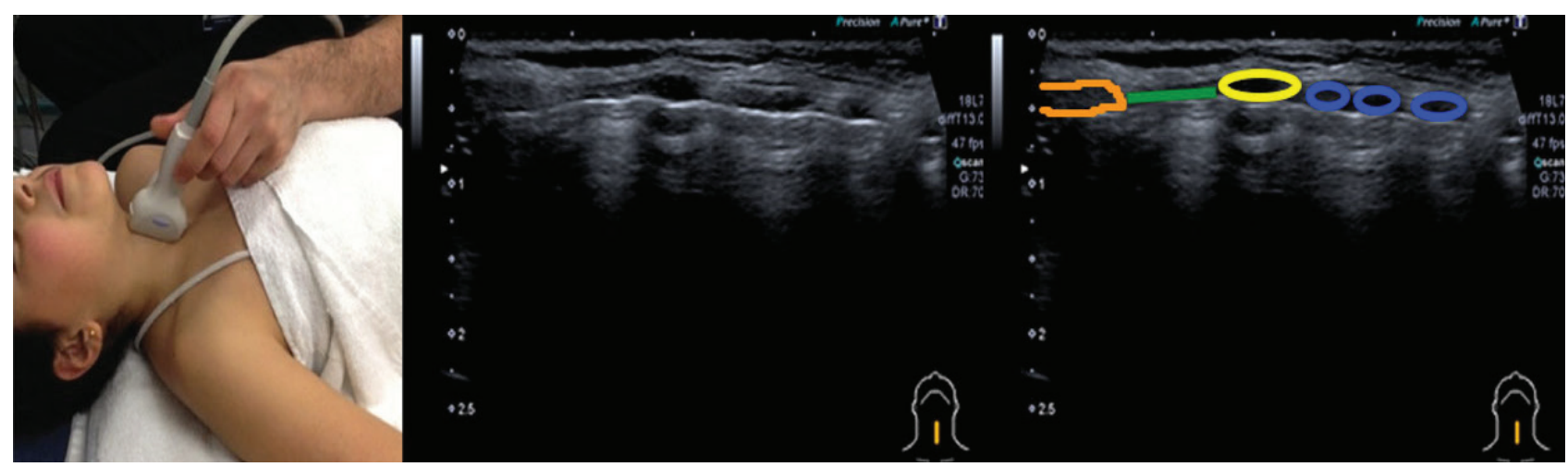

Figure 8. Ultrasound of the longitudinal view of the trachea

Thyroid cartilage (orange), CTM (green), cricoid cartilage (yellow), tracheal rings (blue) ${ }^{29}$ 


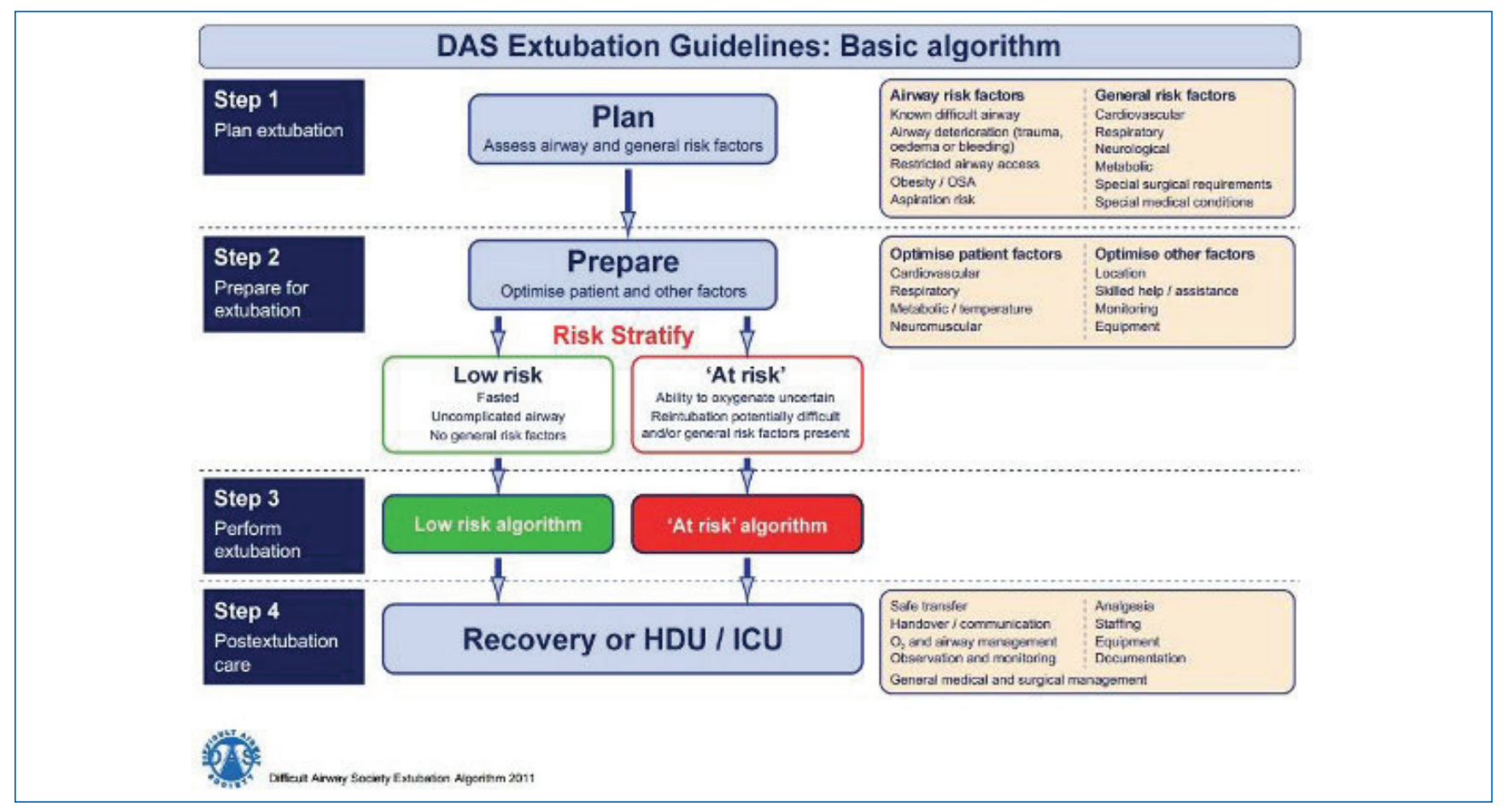

Figure 9. DAS Extubation Guideline

\section{Extubation (plan for the end at the beginning)}

Extubation after a difficult intubation should be performed under defined precautions, optimise your patient prior to extubation. If concerned about airway oedema, consider direct examination of the airway with a laryngoscope or fibreoptic scope. Alternatively, perform a leak test (deflate cuff and listen for leak around tube when patient exhales). Where possible, the patient should be monitored for at least 2-3 hours postoperatively. If there is any doubt a plan for re-intubation should also be in place. ${ }^{4,30}$

Remember re-intubation is often more difficult than at initial attempt due to intubation trauma, surgical insult, intravenous fluids. Figure 9 is the basic algorithm for extubation in adults as suggested by the Difficult Airway Society.

\section{Conclusion}

Local institutional policies on the management of the difficult paediatric airway need to be developed based on the extensive evidence available in adult and paediatric literature. Recognition of the difficult airway, expertise and corresponding infrastructure (team, communication and equipment) are vital in ensuring positive outcomes. The aim should be to plan and prepare in the non-emergency situation, e.g. healthy patients, workshops or simulation, as it is noted that practice develops muscle memory which can be used in the emergency situation. Emphasis must be made on the emerging importance of video laryngoscopy and fibreoptic bronchoscopy in the management of the difficult airway. However, as important are the role of supraglottic airway devices, supplemental oxygenation during attempts and optimal external laryngeal manipulation.

Human error is unavoidable. The onus is on us as practitioners to actively pursue avenues to make these errors less frequent and less damaging.

\section{References}

1. Jimenez N, Posner K, Cheney F, Caplan R, Lee L, Domino K. An Update on Pediatric Anesthesia Liability: A Closed Claims Analysis. Anesth Analg. 2007;104(1).

2. Morray J, Geiduschek J, Caplan R, Posner K, Gild W, Cheney F. A comparison of Pediatric and Adult anesthesia closed malpractice claims. Anesthesiology. 1993;1993(78):461-7.

3. Cote $C$. The difficult paediatric airway. South African Journal of Anaesthesia and Analgesia. 2012;230(18):230-9.

4. Russo S, Becke K. Expected difficult airways in children. Curr Opin Anaesthesiol. 2015;28:321-6.

5. Anesthesiologists ASo. Practice Guidelines for the Management of the Difficult Airway: An updated report. Anesthesiology. 2013;118(2):251-70.

6. Caplan R, Benumof J, Berry F, et al. Practice guidelines for management of the difficult airway. A report by the ASA Task Force on Management of the Difficult Airway. Anesthesiology. 1993;78:597-602.

7. Langeron $O$, Maso E, Huraux C, Guggiari M, Bianchi A, Coriat $P$, et al. Prediction of Difficult Mask Ventilation. Anesthesiology. 2000;92:1229-36.

8. Black A, Flynn P, Smith H, Thomas M, Wilkinson K, Cote C. Development of a guideline for the management of the unanticipated difficult airway in the pediatric practice. Pediatr Anesth. 2015;25:346-62.

9. Paediatric Diffciult Airway Guidelines [Internet]. [Accessed on 15 July 2018]. Available from: https://www.das.uk.com/guidelines/ paediatric-difficult-airway-guidelines

10. Chrimes N. The Vortex: a universal 'high-acuity implementation tool' for emergency airway management. Br J Anaesth. 2016;117(S1):20-7.

11. Difficult airway algorithms and checklists [Internet]. Wordpress. [Accessed on 28 October 2018]. Available from: https://www.ccam.net.au/handbook/ difficult-airway-algorithms-and-checklists/.

12. Cook T, Woodall N, Frek C. Major complications of airway management in the UK: results of the Fourth National Audit Project of the Royal College of Anaesthetists and the Difficult Airway Society. Part 1: Anaesthesia. Br J Anaesth. 2011;105(5):617-31.

13. Engelhardt T, Virag K, Veyckemans F, Habre W. Airway management in paediatric anaesthesia in Europe-insights from the APRICOT (Anaesthesia Practice In Children Observational Trial): a prospective multicentre observational study in 261 hospitals in Europe. Br J Anaesth. 2018;121(1):66-75.

14. Fiadjoe J, Nishisaki A, Jagannathan N, Hunyady A, Greenverg R, Reynolds P, et al. Airway management complications in children with difficult tracheal intubation 
from the Pediatric Difficult Intubation (PeDI) registry: a prospective cohort analysis. Lancet. 2016;4(Jan):37-48.

15. Burjek N, Nishisaki A, Fiadjoe J, Adams D, Peeples K, Raman V, et al. Videolaryngoscopy versus Fiber-optic Intubation through a Supraglottic Airway in Children with a Difficult Airway: An Analysis from the Multicenter Pediatric Difficult Intubation Registry. Anesthesiology. 2017;127(3):432-40.

16. King $M$, Jagannathan $N$. Best practice recommendations for difficult airway management in children - is it time for an update? Br J Anaesth. 2018;121(1):4-7.

17. Jagannathan $\mathrm{N}$, Sohn L, Fiadjoe J. Paediatric difficult airway management: what every anaesthetist should know! Br J Anaesth. 2016;117(1):3-5.

18. 10 Rules for Approaching Difficult Intubation: Always Prepare for Failure [Internet]. 2018 [Accessed on 23 Sept 2019]. Available from: https://www.anesthisiologynews.com/Review-Articles/ Article/08-18/10-Rules-for-Approaching-Difficult-Intubation

19. Fiadjoe J, Litman R, Serber J, Stricker P, Cote C. The Pediatric Airway. In: Cote C, Lerman J, Anderson B, editors. A Practice of Anesthesia for Infants and Children. 6. 6 ed. Philadelphia: Elsevier; 2018. p. 297-339.

20. Cote J, Lerman J, Anderson B. A Practice of Anesthesia for Infants and Children. 6th ed. Elsevier, editor. Philadelphia: Elsevier Inc; 2018.

21. Long E, Cincotta D, Grindlay J, Pellicano A, Clifford M, Sabato S. Implementation of NAP4 emergency airway management recommendations in a quaternarylevel pediatric hospital. Pediatr Anesth. 2017;27:451-60.
22. Intubation checklist: critically ill adults [Internet]. 2017 [Accessed 23 Sept 2018]. Available from: https://das.uk.com/guidelines/icu_guidelines 2017

23. Michiel A, Gray R. Paraglossal straight blade intubation in syndromic children. South African Journal of Anaesthesia and Analgesia. 2018;24(5):118-221.

24. Humphreys $S$, Lee-Archer $P$, Reyne $G$, Long D, Williams T, Schibler A. Transnasal humified rapid insufflation ventilatory exchange (THRIVE) in children: a randomised controlled trial. Br J Anaesth. 2017;118(2):232-8.

25. Beattie C. The Modified Nasal Trumpet Maneuver. Anesth Analg. 2002;95(5):1821.

26. Fiadjoe J, Litman R. Oxygen supplementation during prolonged tracheal intubation should be the standard of care. Br J Anaesth. 2016;117(4):417-8.

27. Sabato $S$, Long E. An institutional approach to the management of the 'Can't Intubate, Can't Oxygenate' emergency in children. Pediatr Anesth. 2016;26:784-93.

28. Okada $Y$, Ishii W, Sato N, Kotani H, liduka R. Management of pediatric 'cannot intubate, cannot oxygenate.' Acute Med Surg. 2017;4:462-6.

29. Stafrace $S$, Engelhardt T, Teoh W, Kristensen M. Essential ultrasound techniques of the pediatric airway. Pediatr Anesth. 2016;26:122-31.

30. Popat M, Mitchell V, Dravid R, Patel A, Swampillai C, Higgs A. Difficult Airway Society Guidelines for the management of tracheal extubation. Anaesthesia. 2012;67:318-40. 